\title{
Impact of sperm DNA chromatin in the clinic
}

\author{
Dimitrios Ioannou $^{1} \cdot$ David Miller $^{2} \cdot$ Darren K. Griffin $^{3} \cdot$ Helen G. Tempest ${ }^{1,4}$
}

Received: 18 November 2015 / Accepted: 22 November 2015 /Published online: 17 December 2015

(C) The Author(s) 2015. This article is published with open access at Springerlink.com

\begin{abstract}
The paternal contribution to fertilization and embryogenesis is frequently overlooked as the spermatozoon is often considered to be a silent vessel whose only function is to safely deliver the paternal genome to the maternal oocyte. In this article, we hope to demonstrate that this perception is far from the truth. Typically, infertile men have been unable to conceive naturally (or through regular IVF), and therefore, a perturbation of the genetic integrity of sperm heads in infertile males has been under-considered. The advent of intracytoplasmic sperm injection (ICSI) however has led to very successful treatment of male factor infertility and subsequent widespread use in IVF clinics worldwide. Until recently, little concern has been raised about the genetic quality of sperm in ICSI patients or the impact genetic aberrations could have on fertility and embryogenesis. This review highlights the importance of chromatin packaging in the sperm nucleus as essential for the establishment and maintenance of a viable pregnancy.
\end{abstract}

Capsule Highlights the importance of chromatin packaging in the sperm nucleus as essential for the establishment and maintenance of a viable pregnancy

Darren K. Griffin

d.k.griffin@kent.ac.uk

1 Department of Human and Molecular Genetics, Herbert Wertheim College of Medicine, Florida International University, Miami, FL, USA

2 Leeds Institute of Genetics, Health and Therapeutics, University of Leeds, Leeds, UK

3 School of Biosciences, University of Kent, Canterbury CT2 7NJ, UK

4 Biomolecular Sciences Institute Florida International University, Miami, FL, USA
Keywords Chromatin · Genetic integrity · Aneuploidy · Sperm DNA fragmentation

\section{Introduction}

Currently, there are limited diagnostic tests available to evaluate the genetic integrity of a semen sample as, traditionally, male fertility evaluation has used conventional semen parameters (concentration, motility morphology, etc.) Although providing fundamental information, it has been strongly suggested that this basic evaluation has questionable clinical value [1]. Within recent years, therefore, several molecular genetic techniques have been developed to assess the genetic integrity of sperm. This review will focus on our current understanding of the genetic integrity of sperm and its impact on fertility and embryogenesis.

The spermatozoon is a highly elaborate and specialized cell that is formed through the process of spermatogenesis, during which a complex cellular program of differentiation occurs. The end result is mature spermatozoa that are essential for reproduction, fertilization, and normal embryo development. The sperm cell is unique in morphology, chromatin structure, and function, and the process of spermatogenesis is characterized by a myriad of changes. Essentially, these can be broken down into three sequential elements: (i) mitotic proliferation (producing large numbers of spermatocytes); (ii) meiotic recombination and chromosome segregation (producing genetically diverse haploid gametes); and (iii) culminates in cytodifferentiation (re-packaging of the haploid genome for delivery to the oocyte). In general, transcription and translation are temporarily disengaged during the post-meiotic stage of spermatogenesis (when round cells are extensively remodeled to form mature spermatozoa) [2]. Furthermore, the storage of mRNAs encoding for protamines is crucial for the completion 
of spermiogenesis [3]. The structure of sperm chromatin and perturbations in its integrity that could have a clinical impact in the form of chromosome aneuploidy, DNA fragmentation, or chromatin organization will form the backbone of this review. This review thus addresses the impact of the unique features of the genetic integrity of sperm in a clinical setting.

\section{Structure of human sperm chromatin}

DNA is, in somatic nuclei complexed, condensed and packaged with a family of proteins (histones) into nucleosomes (chromatin). Histones can be modified by post-translational modifications that regulate the degree of DNA compaction and modulate gene expression by restricting or facilitating access of transcription factors to the DNA. Despite the efficacy of histones in regulating DNA compaction and gene expression, it is clear that an alternative mechanism for DNA packaging has evolved in sperm cells. The haploid sperm chromatin undergoes one of the most significant changes known in biology, in which, the vast majority of histones are replaced with transition proteins, followed by protamines resulting in a highly condensed (typically $10 \%$ or less volume than other somatic cell nucleus) [2], relatively transcriptionally inert cell $[4,5]$. Normal human sperm chromatin remains packaged by $5-15 \%$ of histones $[6,7]$, a higher percentage compared to other mammalian species, e.g., bulls, stallions, hamsters, and mice, which retain $(<5 \%)$ [8]. Therefore, compared to other mammalian species, human sperm chromatin is relatively less compact. Interestingly, dye-binding assays such as aniline blue staining that selectively stain histones are often used as markers of sperm immaturity [9, 10]. As discussed later, this has a bearing on recent reports suggesting an epigenetic role for retained histones (particularly histones carrying post-translational modifications) in sperm.

During chromatin remodeling/compaction in sperm, naturally occurring DNA strand breaks induced by topoisomerase II arise to relieve the torsional stresses that accompany the transition of sperm chromatin from an exclusively nucleosomal to a predominantly protamine-based configuration [11]. Re-packaging with protamines most likely evolved to more efficiently compact chromatin into as small a volume as possible and so facilitate safe delivery of the paternal DNA to the oocyte [2]. Arginine, which is the main component of protamines, is responsible for allowing a higher degree of chromatin compaction by neutralizing the strong negative charges of the phosphate groups in the DNA backbone [12]. Cysteine residues confer extra stability through intermolecular disulfide cross-links [13]. These allow the basic protamine packaging unit (toroid) to be further condensed, with each chromosome becoming a garland of toroids, clustered by centromeres that form a chromo-center in the nuclear interior. The telomeres on the other hand form dimers and tetramers at the nuclear periphery [14-17]. Evidence for regions of the sperm genome remaining associated with nucleosomes that retain a more open conformation comes from studies using salt-washing and/or limited endonuclease digestion of sperm nuclei [18-23]. In two such studies, nucleosome-rich, low saltwashed, and restriction (or micrococcal nuclease) digestionsensitive regions were shown to be associated with important developmental gene sequences involved in embryogenesis $[18,22]$. Other studies suggested that these regions appear to be associated with hypomethylated DNA [24, 25], indicating a potentially permissive state for transcriptional activation of specific gene families involved in early cell differentiation and embryogenesis, perhaps in the early embryo [7]. Altered patterns of sperm DNA and histone methylation have also been reported in infertile men [25], supporting earlier studies into the relationship between DNA methylation and pregnancy outcome in an IVF setting [26]. Additionally, in comparison to other mammalian species (e.g., bull cat, boar, ram) that contain one type of protamine (P1), mouse and human sperm contain two types of protamines (P1 and P2) [27], both present in roughly equal quantities. Improper temporal regulation of these transcripts leading to altered expression of the mature proteins is associated with male infertility especially when the $1: 1$ ratio is perturbed $[27,28]$. Furthermore, an altered P1/P2 ratio has been correlated with a negative impact on embryo quality and IVF outcome although at least one study has shown that a fertility-defining 1:1 ratio is equivocal [29]. As indicated above, infertile men often present with an increased histone to protamine ratio compared to fertile counterparts with a subset of infertile men possessing complete protamine deficiency [30]. In this regard, mouse knockout models in which the intermediate DNA re-packaging transition proteins are lost show impaired fertility and protamine knock-outs are completely infertile [31, 32]. Therefore, the incorrect distribution of histones and protamines throughout the genome could have undesired effects on early embryo development $[2,27]$.

\section{Sperm aneuploidy and its impact in the clinic}

Chromosome aneuploidy (the presence of extra or missing chromosomes in the sperm head) impacts on embryogenesis and the health and development of future offspring. Chromosome aneuploidy is clinically significant, given that it is the leading cause of pregnancy loss and mental retardation in humans. Embryonic aneuploidy can arise from several different mechanisms: (1) a non-disjunction event giving rise to an aneuploid sperm or oocyte, resulting in chromosomally unbalanced gametes or (2) a mitotic loss (e.g., through anaphase lag), gain, or non-disjunction event in the embryo, leading to mosaicism with the presence of normal and aneuploid cells [33]. The parental origin of aneuploidies has been reviewed extensively elsewhere [33-37]. In brief, autosomal trisomies and trisomy $\mathrm{X}$ are typically maternal in origin (70-100 \%). Thus, aneuploidy is predominantly considered to 
be maternal in origin, with advanced maternal age being a significant risk factor for increased risk of aneuploidy [33]. However, it should be noted that a paternal contribution remains for the aforementioned trisomies. Additionally, certain clinically viable aneuploidies (e.g., monosomy X, 47, XXY, and $47, \mathrm{XYY}$ ) are predominantly paternal in origin with a prevalence of $70-80,50$, and $100 \%$, respectively [38]. Advancing paternal age has less impact on chromosome aneuploidy with only a slight increased risk emerging for certain chromosomes [39].

Sperm aneuploidy can be readily identified by either sperm karyotyping (achieved after fertilization of a human sperm with a hamster oocyte) or by interphase fluorescence in situ hybridization (FISH) in human sperm nuclei. Early sperm aneuploidy studies studied sperm karyotypes, a technically demanding and labor-intensive method that could only study a relatively low number of sperm cells from each individual, that were capable of fertilizing a hamster oocyte. The major benefit of this method is that it allows both numerical and structural chromosome aberrations to be identified. The advent of FISH revolutionized our ability to study sperm aneuploidy allowing thousands of sperm to be studied and was rapidly adopted as a research tool to investigate the paternal contribution to aneuploidy. Early cumulative data from the sperm karyotype studies revealed a higher incidence of structural chromosome compared to numerical aberrations within sperm cells (6-7 \% versus $1-2 \%$, respectively) $[34,40]$. However, this is based on a relatively small number of karyotypes and subjects. Therefore, for the remainder of this section, we will focus on FISH studies. Several comprehensive reviews have been published compiling previous studies evaluating sperm aneuploidy levels in fertile and infertile patient cohorts for all chromosomes [38, 41-43]. Taken together, the vast majority of studies have demonstrated that all men produce a proportion of aneuploid sperm. However, an increased percentage of sperm aneuploidy has been significantly associated with reduced semen parameters and shown to increase with the severity of the male factor infertility [38, 41-43]. Furthermore, specific chromosomes namely chromosomes 21, 22 and $\mathrm{X}$, and Y typically display a two- to threefold increase in chromosome non-disjunction compared to the rest of the chromosome complement [38, 41-43]. This is perhaps not surprising as meiotic recombination plays a crucial role in keeping homologous chromosomes together. The aforementioned chromosomes have the smallest pairing regions and usually only present with single chiasmata $[40,44,45]$.

The emerging sperm aneuploidy picture from studying males with different types of infertility (e.g., oligo-, astheno-, terato-, zoospermia) is that they have on average of threefold increase with a two- to tenfold higher prevalence of numerical chromosome abnormalities compared to their fertile counterparts [44, 46]. Patients with more severe morphological abnormalities in sperm (e.g., macrocephalic-multi-flagellated sperm, globozoospermia) manifest $10-30 \times$ and $8-10 \times$ increase in aneuploidy, respectively, compared to controls [38, 47, 48]. Patients with oligoasthenoteratozoospermia (OAT) have been studied extensively using FISH probes by many groups, and an increased level (up to 30-fold) of aneuploidies (disomy, diploidy, nullisomy) has been found for all investigated chromosomes compared to their fertile counterparts [42, 46, 47, 49-56].

In terms of sperm aneuploidy in individuals with numerical or structural chromosome aberrations (e.g., 47,XXY, 47,XYY, Robertsonian, or reciprocal translocations) it appears that the actual observed rates are lower from the theoretical numbers expected from the behavior of the meiotic trivalents or quadrivalents possibly due to some selection against these aneuploid sperm (e.g., an unknown meiotic checkpoint) [38, 47, $48,56,57]$. Non-mosaic Klinefelter patients are reported to have an average of $6 \%$ sex chromosome aneuploidy within their sperm [47]. The proportion of unbalanced gametes increases with structural chromosome aberrations with carriers of reciprocal and Robertsonian translocations demonstrating a 50 and $15 \%$ frequency of unbalanced gametes, respectively [38]. Finally, patients with Y microdeletions also appear to have a small but significant increased risk for sex chromosome aneuploidies [58, 59].

Therefore, although the majority of embryonic aneuploidy is of maternal origin, the sperm genome has a significant role in the formation of a euploid embryo and thus the development of a healthy offspring. As discussed above, in cases of male factor infertility, there is an increased risk for transmitting an aneuploid paternal genome to the oocyte. IVF centers in the UK were surveyed as to whether they routinely perform sperm aneuploidy screening and if they perceive there to be a genetic risk to offspring conceived by intracytoplasmic sperm injection (ICSI) [60]. The vast majority indicated that while their center rarely performed such screening, there was merit in doing so [60]. This opinion gains some weight given that studies to date suggest that there is little to no evidence that aneuploid sperm are at any disadvantage in fertilizing an oocyte compared to a haploid sperm $[47,48,56]$. A handful of studies have convincingly demonstrated a distinct lack of selection against chromosomally abnormal sperm. These studies provide evidence to suggest that increased sperm aneuploidy translates to increased aneuploidy in embryos [61]. The approximate threefold increase in sperm aneuploidy observed in infertile populations is mirrored by a threefold increase in de novo chromosomal abnormalities in children born after ICSI [62]. In the case of chromosomal translocations, the high percentage of chromosomally unbalanced sperm is shown to translate to a high proportion of chromosomally unbalanced embryos [63]. It is clear that certain individuals have an increased risk of producing high levels of sperm aneuploidy including the following: infertile patients (particularly OAT, non-obstructive azoospermia) and patients with structural and numerical chromosome aberrations $[38,47,48,56]$. 


\section{Sperm DNA fragmentation and its impact in the clinic}

The need to identify novel markers that can better discriminate between fertile and infertile men and can assess the genetic integrity has given rise to the field of sperm DNA fragmentation. In recent years, this has been of particular interest since with the advent of assisted reproduction technologies, genetic defects that can be transmitted as natural selection barriers to fertilization are bypassed [30]. The effect of abnormal sperm chromatin on subsequent development will depend on the severity of the damage and the repair capacity of the oocyte.

The etiology of sperm DNA damage which is characterized by single- and double-break strands (SSBs and DSBs) is multifactorial and can be related to intrinsic and extrinsic factors. Intrinsic factors include the following: protamine deficiency, excessive reactive oxygen species (ROS) levels, and abortive apoptosis; extrinsic factors include the following: environmental exposures, chemotherapy, and possibly lifestyle factors $[8,64,65]$. As discussed previously during the chromatin remodeling process in sperm, naturally occurring DNA strand breaks occur to facilitate the replacement of histones by protamines. Once chromatin is repackaged, these breaks are subsequently resealed [11]. Perturbations within this machinery of break and repair can cause altered chromatin compaction and residual breaks in the sperm DNA [11] that can result in measurable DNA fragmentation in the ejaculate.

Sperm DNA damage has also been associated with high levels of ROS detected in the semen of approximately $25 \%$ of infertile men [64]. The susceptibility to ROS damage stems from the presence of unsaturated fatty acids in the plasma membrane, necessary for membrane fluidity which is required in the acrosome reaction during fertilization [66]. The only defence mechanism against ROS is the antioxidant ability of the seminal plasma and the sperm chromatin compactness [11]. However, free radicals can be produced both by defective spermatozoa and semen leukocytes thus inducing sperm damage and conferring male subfertility $[11,64,66]$. The window of time during which DNA damage occurs is still under debate but it most likely occurs during the epididymal maturation as this is the period during which spermatozoa are most exposed to ROS [11].

Abortive apoptosis has been postulated as another theory for DNA damage and has been associated with a form of selective apoptosis that, under normal conditions, regulates the production of abnormal sperm in spermatogenesis and limits the population of germ cells to a number that can be supported by the Sertoli cells [11, 64, 67]. Over-expression of this process could lead to oligo- or azoospermia whereas under-expression could give rise to a high proportion of abnormal sperm, which could impair fertilization [67]. Using a marker for apoptosis (Fas), it was found that less than $10 \%$ of apoptotic sperm exist in normozoospermic men whereas approximately $60 \%$ of oligospermic men have more than $10 \%$ of apoptotic sperm [67]. However, other studies have not found this correlation so the jury for the definitive association of DNA fragmentation and apoptotic biomarkers remains elusive $[8,30]$.

In terms of extrinsic factors, exposure to environmental pollutants (e.g., pesticides and pollution) has also been associated with DNA damage [65, 68]. Chemotherapy treatment in males of reproductive age has been linked with impaired spermatogenesis, increased sperm aneuploidy levels, and increased DNA fragmentation [69, 70]. For the most part, studies have demonstrated that following chemotherapy, recovery of spermatogenesis and return to baseline aneuploidy levels may occur months to years after treatment has ceased [70]. However, DNA damage induced seems to be more persistent than numerical chromosome defects $[8,64,69]$. The impact of lifestyle factors should not be neglected either, since obesity, smoking, and certain occupations (e.g., welding, baking) have been associated with decreased semen quality and increased levels of DNA damage [30, 67, 71].

The widespread use of ICSI and a desire to improve its success rates have led to the incentive to develop assays to test the genetic integrity of sperm. These assays have been developed in an effort to measure sperm chromatin damage, aid in the diagnosis of male infertility, and provide predictive reproductive outcomes. It is beyond the scope of this chapter to review these tests in detail; however, they can be summarized in three groups: (1) sperm chromatin structural probes (e.g., chromomycin $A_{3}$, sperm chromatin structural assaySCSA); (2) tests that directly assess DNA fragmentation (e.g., TUNEL, COMET assays); and (3) sperm nuclear matrix assays (e.g., sperm nuclear matrix stability assay, chromatin dispersion test) [8]. Several review articles provide more detailed description of each technique with principles, detection methods, and pros and cons of each approach $[8,11,72,73]$.

In order to evaluate the clinical value of the sperm DNA fragmentation tests, it is important to assess the relationship of these tests with pregnancy outcomes. Detailed meta-analyses of published studies have been previously published and will be discussed briefly $[8,30,73]$. A small number of studies have shown that when DNA fragmentation exceeds $30 \%$ with the SCSA test, it indicates a lower likelihood/close to zero probability for fertilization through natural pregnancies and intrauterine inseminations (IUIs) $[8,74]$. In terms of IVF, the cohort of studies is quite heterogeneous $(n>20)$ and the trend seems to indicate that lower IVF pregnancy rates is correlated with increased sperm DNA fragmentation. When IVF followed by ICSI comes to the equation, studies suggest surprisingly that level of DNA damage appears not to significantly impact ICSI pregnancies, but this can be attributed to the careful selection of sperm and embryo following ICSI. This selection process likely abrogates the adverse effects of sperm DNA damage. When the DNA fragmentation index is assessed by SCSA, a predictive threshold of $27 \%$ is required for a 
successful pregnancy via IVF and/or ICSI. However, other groups have demonstrated that pregnancies can occur after ICSI utilizing sperm with a much high proportion of DNA damage [8]. This underlines the requirement for long-term follow-up studies to evaluate any consequences on pregnancies following ICSI utilizing sperm with high levels of sperm DNA damage. It should be noted that studies have also correlated pregnancy loss after IVF or ICSI with high levels of sperm DNA damage [73]. High levels of DNA fragmentation may be unlikely to directly affect fertilization rates since the embryonic genome starts expression at the 4-8 cell stage. Therefore, sperm samples with high levels of DNA damage could have more clinical ramifications during the later stages of embryonic development (i.e., blastocyst), which may in part explain its association with pregnancy loss [73].

A consensus exists within the community that increased sperm DNA fragmentation is associated with a lower chance of conceiving through natural, IUI, or IVF, but not ICSI methods. Current research suggests that couples who may benefit most from assessment of sperm DNA fragmentation are couples with recurrent miscarriages [73] or unexplained male factor infertility [8]. However, the role and impact of sperm DNA fragmentation on fertilization, embryogenesis, and development remains rudimentary at best due to the relatively small number of studies and heterogeneous findings $[8$, $30,73]$. More studies are needed to better understand the etiology of sperm DNA fragmentation, its potential association with increased risk of pregnancy loss, and identification of the optimal assays. It is clear that these studies are required to further validate the clinical significance of sperm DNA damage before assessment of sperm DNA fragmentation is a routine test in andrology labs and patient management.

\section{Chromatin organization and modification-the "missing" links with the clinic?}

Previously, we have discussed the competence aspects of sperm "chromatin" either in the form of chromosome constitution (aneuploidy) or DNA damage and the impact that their assessment has in the clinic. Such tests have been developed in an effort to provide patients and clinicians with improved diagnostic methods for infertile patient and to better predict ART outcomes. We can thus postulate that a sperm head with the correct chromosome copy number and absence of DNA damage is a "healthy" gamete with increased potential for the faithful transfer from the paternal genome and epigenetic input to the zygote for the development of healthy offspring. Other markers that can be considered important for nuclear health and normal cellular function are the appropriate spatiotemporal organization of the chromatin (i.e., the position of chromosomes and/or genes within the nucleus) and the epigenetic marks that the chromatin carries into the egg.
Chromosomes occupy distinct non-random positions in most interphase nuclei (termed chromosome territoriesCTs) [75-80]. This organization appears to be evolutionary conserved, and two models ("gene density related" and "chromosome size related") have been used to describe the position of chromosomes in different somatic cell types [81]. Although the functional implication of nuclear organization is still an active topic of discussion and beyond the main scope of this chapter, the feature of the distinct non-random position, the evolutionary conservation, and the changes in the patterns of organization observed in certain disease conditions (e.g., laminopathies, Hutchinson-Gilford progeria, breast cancer) [82] highlights the importance for the maintenance of stable architecture for proper cellular function [83].

Any perturbation in nuclear architecture could induce change in the local gene environment and availability to transcription factors leading to possible mis-regulation or failure to take part in transcription [84]. It has been hypothesized that chromatin organization may be crucial for spatial chromatin differentiation, modifications of the epigenome transmitted to the embryo, and normal embryogenesis which may have evolved with other mammalian regulatory systems including genomic imprinting and $\mathrm{X}$ inactivation $[85,86]$. Thus, the study of nuclear organization in the male gamete could have important ramifications for early embryogenesis when we take into account the unique features of the haploid sperm DNA packaging. Although we have a good understanding of the different chromatin packaging in sperm compared to other cell types, our understanding of the organization of chromatin in spermatogenesis is poor [87].

The vast majority of studies published in this area have been focused solely on the organization of the mature haploid, protamine packaged sperm cell [14, 16, 88-96]. Data emerging from these studies suggest that organization of the human sperm nucleus is different than that of the somatic cells. Chromosomes appear to be clustered via their centromeres to form a chromo-center in the interior of the nucleus, while the telomeres are preferentially located toward the nuclear periphery where they form dimers and tetramers [14-17, 95]. The chromo-center appears to be formed by pericentric heterochromatin from different chromosomes that have a tendency to aggregate [15,92]. A similar spatial organization has been shown to be evolutionarily conserved in other mammals (mouse [89, 97], bovine, pig, horse, and rat) [95]. The organization of CTs or specific chromosomal regions has been addressed both radially (i.e., location in relation to the nuclear interior to the periphery) and in some studies longitudinally (i.e., distribution in relation to the sperm head and tail) [94]. The hypothesized functional implication of the longitudinal and radial distribution of chromosomes in human sperm is thought to be related to the ordered exodus after fertilization. Thus, the order that the maternal cytoplasmic environment encounters the paternal genome [98] and potentially remodels 
and repairs DNA damage on the early exiting chromosomes may be important compared to the late exiting regions of the genome. It should also be emphasized that the positions of the sex chromosomes relative to the acrosome are similar in sperm of all mammals (but not birds), implicating a functional significance [86].

Assuming a functional significance for the non-random organization of chromosomes in human sperm with its possible impact in fertilization, it is reasonable to suggest that altered nuclear organization could be a measurable phenotype in the sperm of infertile men [91] and could provide an additional explanation for idiopathic infertility. Early evidence for a clinical impact of chromosome organization in sperm came from observations that sperm used in ICSI which had not undergone the acrosome reaction, showed impaired decondensation of apically located chromatin [16]. Furthermore, this delay in decondensation was observed to hinder the progression of the first mitotic division of the zygote and has provided indirect evidence to potentially explain the increase in sex chromosome aneuploidies observed in offspring after ICSI. This provides a tentative link with the more firmly established clinical impact of sperm aneuploidy and DNA fragmentation in nuclear health. It has been proposed that some infertile men may have a different category of sperm chromatin abnormality related to atypical packing of CTs in sperm, aberrant positioning of chromosomes, or disturbed telomere-centromere interactions [98]. Currently, the proposed relationship between male infertility and altered nuclear organization remains hypothetical, with direct evidence to prove or disprove this link being somewhat lacking. Indeed, only a handful of studies have tried to address this link [88, 92,99-102]. Currently, we are investigating the 2D and 3D nuclear organization (both radially and longitudinally) of chromosomes within human sperm [94]. Additionally, we are investigating sub-chromosomal structures (telomeres, centromeres), imprinted genes, and important developmental genes that have been shown to retain histones and unique methylation patterns in the protamine packaged sperm. It is clear that the sperm cell can no longer be considered a vessel for delivering a silent genome but is rather an epigenetically poised cell that is crucial for fertilization and embryogenesis.

\section{Evidence for an epigenetic signature in sperm chromatin that could affect embryogenesis}

As indicated above, differential methylation of sperm DNA and the deregulated presence of nucleosomes on embryologically important gene loci appear to correspond with an infertile phenotype $[25,26]$. These data suggest the possibility that in addition to the genome itself, sperm chromatin carries an epigenetic 'signature' to the egg potentially borne on modified histones. Histone modifications are numerous and beyond the scope of this review [2], but because of the known association of lysine (K)-modified $\mathrm{H} 3$ with expression permissive (H3K36me3) and restrictive (H3K27me3) chromatin domains, reports on differential lysine methylation in sperm chromatin are particularly intriguing [7, 25]. These studies follow a raft of earlier reports demonstrating histones in mature sperm, the earliest of which was with CENPA in bull sperm [103] and reviewed in [2, 27]. These reports include microscopic evidence for the transmission and survival to syngamy of paternal nucleosomes. Moreover, biochemical evidence for the transmission and retention of structurally unusual (smaller) sperm nucleosomes containing sperm-specific histone variants (H2AL1 and H2AL2) has also been put forward [104] and potentially confirmed by proteomic analysis [105]. Sperm histones may play an essential role in promoting normal embryogenesis although evidence to date suggests that neither sperm-specific H2AL isoforms, nor any other histone modification persists for long following fertilization [106, 107]. Moreover, as the successful generation of (murine) gynogenic parthenotes following alteration of the H19/Igf2 imprinted locus demonstrates [108], the paternal genome is actually dispensable altogether with respect to embryonic development. It is more likely, therefore, that an epigenetic signature borne on sperm nucleosomes performs more spermcentric functions that may nevertheless be prone to deregulation in infertile men, perhaps by aberrant histone deposition as reported elsewhere $[25,109]$. In this regard, the essential role of a testis-specific form of the double bromodomain containing BET family, BRDT in facilitating chromatin reorganization during spermiogenesis should be considered, particularly in view of a potential role for this factor in infertility [110, 111]. BRDT is involved in both the formation of the sperm chromo-centre and in the regulation of translational control of stored mRNPs, both vital functions for fertility. On the other hand, the case for some form of histone-based paternal epigenetic contribution is supported by reports that differentially condensed blocks of chromatin containing developmentally important gene sequences analogous to the nucleosomeenriched regions of mammalian sperm chromatin. These are also found in the zebra fish, which does not use protamine to repackage its genome [112]. Furthermore, two other reports have shown that the DNA methylome of the zebra fish egg is fully reprogrammed to resemble the incoming sperm methylome shortly after fertilization [113, 114]. Interestingly, a link between gamete/embryo DNA methylation dynamics and a post-fertilization function for (human) sperm histones was reported earlier in a study showing that DNA methylation-free regions in the early embryo correspond with nucleosome-rich regions in sperm chromatin [115]. These studies and the findings from Hammoud et al. [7] provide supportive evidence of a role for nucleosomal, probably euchromatic regions of the incoming paternal genome with subsequent DNA methylation patterns in the early embryo. However, as the example of gynogenic parthenotes strongly suggests, the 
sperm's epigenetic influence is not a prerequisite for subsequent and successful embryonic development.

An alternative possibility is that modified sperm histones introduced into the oocyte on fertilization provide an essential role in facilitating the sperm's 'acceptance' by the egg as a complementary agent. Such a hypothesis is not so outlandish when considering the risk the sperm poses to the oocyte as an invasive cell. Ideas of 'confrontation,' 'recognition,' and 'consolidation' have already been put forward in relation to the potential introduction and management of potentially harmful parasitic mobile elements [116] and also 'tolerance' of sexskewing bacterial endosymbionts such as Wolbachia [117, 118]. Such a hypothesis is fully compatible with roles for sperm histones, modified or not, and of course, paternal DNA methylation in male fertility without over stating their importance in the support of embryogenesis per se. The apparent preferential localization of sperm histones to the exome, confirmed in independent studies [7, 18, 119], is likely part of the matching process required for successful syngamy (a particularly attractive notion assuming nucleosomal stretches of sperm chromatin have more immediate access to maternal factors at fertilization than protamine-bound regions). This adds further importance to the potential role of nuclear organization as this may function as an additional layer of epigenetic regulation. Such proposed studies will ultimately identify the spatio-temporal localization of targeted genes throughout spermatogenesis and identify whether nuclear organization is perturbed in infertile men. This field remains an active area of research with possible ramifications for improved screening (in combination with standard tests), diagnosis, and predictions of ART treatment efficacy.

\section{Conclusions}

It is self-evident that the paternal genome is critical for the promotion of normal fertilization and embryogenesis and with infertility affecting approximately one in six couples of the western world and male factor contributing to around $50 \%$ of cases, there is an unequivocal need for further research into the male gamete. Understanding the role(s) played by the sperm's unique and specialized chromatin structure in conferring a fertile phenotype is also preferable, and the advent of ART makes the evaluation and impact of sperm chromatin structure all the more important. The ultimate goal is the development of rapid reliable tests that can assess the genomic integrity of sperm to be used in ART and to identify novel aspects of chromatin integrity (e.g., genome organization) that may play a crucial role in fertilization and early embryogenesis. The development of such tests outlined in this chapter aims to further our understanding of paternal contribution and requirements for normal fertilization and embryogenesis. The goal is to create an "arsenal" of analytical tools to better diagnose and assess the genetic integrity of the paternal genome to facilitate the transfer of the single euploid embryo. Undoubtedly, the development of reliable tools to assess the integrity of the paternal genome will assist andrologists, embryologists, clinicians, and couples undertaking ART to allow more informed decisions to be taken regarding their reproductive choices.

Open Access This article is distributed under the terms of the Creative Commons Attribution 4.0 International License (http:// creativecommons.org/licenses/by/4.0/), which permits unrestricted use, distribution, and reproduction in any medium, provided you give appropriate credit to the original author(s) and the source, provide a link to the Creative Commons license, and indicate if changes were made.

\section{References}

1. Lewis SE. Is sperm evaluation useful in predicting human fertility? Reproduction. 2007;134:31-40.

2. Miller D, Brinkworth M, Iles D. Paternal DNA packaging in spermatozoa: more than the sum of its parts? DNA, histones, protamines and epigenetics. Reproduction. 2010;139(2):287-301.

3. Rathke $\mathrm{C}$ et al. Chromatin dynamics during spermiogenesis. Biochim Biophys Acta. 2014;1839(3):155-68.

4. Li Y et al. Characterization of nucleohistone and nucleoprotamine components in the mature human sperm nucleus. Asian J Androl. 2008;10(4):535-41.

5. McLay DW, Clarke HJ. Remodelling the paternal chromatin at fertilization in mammals. Reproduction. 2003;125(5):625-33.

6. Gatewood JM et al. Isolation of 4 core histones from human sperm chromatin representing a minor subset of somatic histones. J Biol Chem. 1990;265(33):20662-6.

7. Hammoud SS et al. Distinctive chromatin in human sperm packages genes for embryo development. Nature. 2009;460(7254): 473-8.

8. Erenpreiss $\mathrm{J}$ et al. Sperm chromatin structure and male fertility: biological and clinical aspects. Asian J Androl. 2006;8(1):11-29.

9. Ovari L et al. Double probing individual human spermatozoa: aniline blue staining for persistent histones and fluorescence in situ hybridization for aneuploidies. Fertil Steril. 2009;93(7): 2255-61.

10. Sati L, Huszar G. Methodology of aniline blue staining of chromatin and the assessment of the associated nuclear and cytoplasmic attributes in human sperm. Methods Mol Biol. 2013;927:425-36.

11. Tarozzi $\mathrm{N}$ et al. Clinical relevance of sperm DNA damage in assisted reproduction. Reprod Biomed Online. 2007;14(6):74657.

12. Bjorndahl L, Kvist U. Human sperm chromatin stabilization-a proposed model including zinc bridges. Mol Hum Reprod. 2010;16(1):23-9.

13. Ward WS. Function of sperm chromatin structural elements in fertilization and development. Mol Hum Reprod. 2010;16(1): $30-6$.

14. Zalensky AO et al. Well-defined genome architecture in the human sperm nucleus. Chromosoma. 1995;103(9):577-90.

15. Zalensky AO et al. Organization of centromeres in the decondensed nuclei of mature human sperm. Chromosoma. 1993;102(8):509-18. 
16. Luetjens CM, Payne C, Schatten G. Non-random chromosome positioning in human sperm and sex chromosome anomalies following intracytoplasmic sperm injection. Lancet. 1999;353(9160): 1240.

17. Solov'eva L et al. Nature of telomere dimers and chromosome looping in human spermatozoa. Chromosome Res. 2004;12(8): 817-23.

18. Arpanahi A et al. Endonuclease-sensitive regions of human spermatozoal chromatin are highly enriched in promoter and CTCF binding sequences. Genome Res. 2009;19(8):1338-49.

19. Gardiner-Garden $\mathrm{M}$ et al. Histone- and protamine-DNA association: conservation of different patterns within the beta-globin domain in human sperm. Mol Cell Biol. 1998;18(6):3350-6.

20. Gatewood JM et al. Sequence-specific packaging of DNA in human sperm chromatin. Science. 1987;236(4804):962-4.

21. Henikoff $\mathrm{S}$ et al. Genome-wide profiling of salt fractions maps physical properties of chromatin. Genome Res. 2009;19(3):460-9.

22. Saida $\mathrm{M}$ et al. Key gene regulatory sequences with distinctive ontological signatures associate with differentially endonuclease accessible mouse sperm chromatin. Reproduction. 2011;142(1): $73-86$.

23. Wykes SM, Krawetz SA. The structural organization of sperm chromatin. J Biol Chem. 2003;278(32):29471-7.

24. Molaro A et al. Sperm methylation profiles reveal features of epigenetic inheritance and evolution in primates. Cell. 2011;146(6):1029-41.

25. Hammoud SS et al. Genome-wide analysis identifies changes in histone retention and epigenetic modifications at developmental and imprinted gene loci in the sperm of infertile men. Hum Reprod. 2011;26(9):2558-69.

26. Benchaib $M$ et al. Influence of global sperm DNA methylation on IVF results. Hum Reprod. 2005;20(3):768-73.

27. Carrell DT, Hammoud SS. The human sperm epigenome and its potential role in embryonic development. Mol Hum Reprod. 2010;16(1):37-47.

28. Aoki VW, Liu LH, Carrell DT. Identification and evaluation of a novel sperm protamine abnormality in a population of infertile males. Hum Reprod. 2005;20(5):1298-306.

29. Nanassy L et al. The clinical utility of the protamine 1/protamine 2 ratio in sperm. Protein Pept Lett. 2011;18(8):772-7.

30. Zini A, Sigman M. Are tests of sperm DNA damage clinically useful? Pros and cons. J Androl. 2009;30(3):219-29.

31. Adham IM et al. Teratozoospermia in mice lacking the transition protein 2 (Tnp2). Mol Hum Reprod. 2001;7(6):513-20.

32. Shirley $\mathrm{CR}$ et al. Abnormalities and reduced reproductive potential of sperm from Tnp1- and Tnp2-null double mutant mice. Biol Reprod. 2004;71(4):1220-9.

33. Hassold T, Hunt P. To err (meiotically) is human: the genesis of human aneuploidy. Nat Rev Genet. 2001;2(4):280-91.

34. Hassold T et al. Human aneuploidy: incidence, origin, and etiology. Environ Mol Mutagen. 1996;28(3):167-75.

35. Hassold T, Hall H, Hunt $P$. The origin of human aneuploidy: where we have been, where we are going. Hum Mol Genet. 2007;16(Spec No 2):R203-8.

36. Hassold T, Hunt P. Maternal age and chromosomally abnormal pregnancies: what we know and what we wish we knew. Curr Opin Pediatr. 2009;21(6):703-8

37. Nagaoka SI, Hassold TJ, Hunt PA. Human aneuploidy: mechanisms and new insights into an age-old problem. Nat Rev Genet. 2012;13(7):493-504

38. Templado C, Vidal F, Estop A. Aneuploidy in human spermatozoa. Cytogenet Genome Res. 2011;133(2-4):91-9.

39. Fonseka KG, Griffin DK. Is there a paternal age effect for aneuploidy? Cytogenet Genome Res. 2011;133(2-4):280-91.

40. Martin RH. Meiotic errors in human oogenesis and spermatogenesis. Reprod Biomed Online. 2008;16(4):523-31.
41. Shi Q, Martin RH. Aneuploidy in human sperm: a review of the frequency and distribution of aneuploidy, effects of donor age and lifestyle factors. Cytogenet Cell Genet. 2000;90(3-4):219-26.

42. Tempest HG, Griffin DK. The relationship between male infertility and increased levels of sperm disomy. Cytogenet Genome Res. 2004;107(1-2):83-94.

43. Templado C, Uroz L, Estop A. New insights on the origin and relevance of aneuploidy in human spermatozoa. Mol Hum Reprod. 2013;19(10):634-43.

44. Martin RH. Mechanisms of nondisjunction in human spermatogenesis. Cytogenet Genome Res. 2005;111(3-4):245-9.

45. Martin RH. Meiotic chromosome abnormalities in human spermatogenesis. Reprod Toxicol. 2006;22(2):142-7.

46. Tempest HG et al. The association between male infertility and sperm disomy: evidence for variation in disomy levels among individuals and a correlation between particular semen parameters and disomy of specific chromosome pairs. Reprod Biol Endocrinol. 2004;2:82.

47. Tempest HG. Meiotic recombination errors, the origin of sperm aneuploidy and clinical recommendations. Syst Biol Reprod Med. 2011;57(1-2):93-101.

48. Harton GL, Tempest HG. Chromosomal disorders and male infertility. Asian J Androl. 2012;14(1):32-9.

49. Bernardini $L$ et al. Comparison of gonosomal aneuploidy in spermatozoa of normal fertile men and those with severe male factor detected by in-situ hybridization. Mol Hum Reprod. 1997;3(5): 431-8.

50. Pang MG et al. Detection of aneuploidy for chromosomes 4, 6, 7, 8, $9,10,11,12,13,17,18,21, \mathrm{X}$ and $\mathrm{Y}$ by fluorescence in-situ hybridization in spermatozoa from nine patients with oligoasthenoteratozoospermia undergoing intracytoplasmic sperm injection. Hum Reprod. 1999;14(5):1266-73.

51. Pfeffer J et al. Aneuploidy frequencies in semen fractions from ten oligoasthenoteratozoospermic patients donating sperm for intracytoplasmic sperm injection. Fertil Steril. 1999;72(3):472-8.

52. Durakbasi-Dursun HG et al. A new approach to chromosomal abnormalities in sperm from patients with oligoasthenoteratozoospermia: detection of double aneuploidy in addition to single aneuploidy and diploidy by five-color fluorescence in situ hybridization using one probe set. Fertil Steril. 2008;89(6):1709-17.

53. Storeng RT et al. Incidence of sex chromosome abnormalities in spermatozoa from patients entering an IVF or ICSI protocol. Acta Obstet Gynecol Scand. 1998;77(2):191-7.

54. Ushijima $\mathrm{C}$ et al. Analysis of chromosomal abnormalities in human spermatozoa using multi-colour fluorescence in-situ hybridization. Hum Reprod. 2000;15(5):1107-11.

55. Zhang QF, Lu GX. Investigation of the frequency of chromosomal aneuploidy using triple fluorescence in situ hybridization in 12 Chinese infertile men. Chin Med J (Engl). 2004;117(4):503-6.

56. Hann MC, Lau PE, Tempest HG. Meiotic recombination and male infertility: from basic science to clinical reality? Asian J Androl. 2011;13(2):212-8.

57. Templado C, Bosch M, Benet J. Frequency and distribution of chromosome abnormalities in human spermatozoa. Cytogenet Genome Res. 2005;111(3-4):199-205.

58. Shah K et al. The genetic basis of infertility. Reproduction. 2003;126(1):13-25.

59. O'Flynn O' Brien KL, Varghese AC, Agarwal A. The genetic causes of male factor infertility: a review. Fertil Steril. 2010;93(1):1-12.

60. Griffin DK et al. Safety issues in assisted reproduction technology: should men undergoing ICSI be screened for chromosome abnormalities in their sperm? Hum Reprod. 2003;18(2):229-35.

61. Gianaroli L, Magli MC, Ferraretti AP. Sperm and blastomere aneuploidy detection in reproductive genetics and medicine. $\mathrm{J}$ Histochem Cytochem. 2005;53(3):261-7. 
62. Van Steirteghem A et al. Follow-up of children born after ICSI. Hum Reprod Update. 2002;8(2):111-6.

63. Escudero $\mathrm{T}$ et al. Predictive value of sperm fluorescence in situ hybridization analysis on the outcome of preimplantation genetic diagnosis for translocations. Fertil Steril. 2003;79 Suppl 3:1528-34.

64. Zini A, Libman J. Sperm DNA damage: clinical significance in the era of assisted reproduction. CMAJ. 2006;175(5):495-500.

65. Aitken RJ, De Iuliis GN. Origins and consequences of DNA damage in male germ cells. Reprod Biomed Online. 2007;14(6):72733.

66. Aitken RJ, De Iuliis GN. On the possible origins of DNA damage in human spermatozoa. Mol Hum Reprod. 2010;16(1):3-13.

67. Varghese AC, du Plessis SS, Agarwal A. Male gamete survival at stake: causes and solutions. Reprod Biomed Online. 2008;17(6): 866-80.

68. Barratt CL et al. Sperm DNA: organization, protection and vulnerability: from basic science to clinical applications - a position report. Hum Reprod. 2010;25(4):824-38.

69. O'Flaherty $\mathrm{C}$ et al. Impact of chemotherapeutics and advanced testicular cancer or Hodgkin lymphoma on sperm deoxyribonucleic acid integrity. Fertil Steril. 2010;94(4):1374-9.

70. Tempest $\mathrm{HG}$ et al. Sperm aneuploidy frequencies analysed before and after chemotherapy in testicular cancer and Hodgkin's lymphoma patients. Hum Reprod. 2008;23(2):251-8.

71. Calogero A et al. Cigarette smoke extract immobilizes human spermatozoa and induces sperm apoptosis. RBM Online. 2009;19(4):564-71.

72. Aitken RJ, Koppers AJ. Apoptosis and DNA damage in human spermatozoa. Asian J Androl. 2011;13(1):36-42.

73. Zini A. Are sperm chromatin and DNA defects relevant in the clinic? Syst Biol Reprod Med. 2011;57(1-2):78-85.

74. Ruvolo $G$ et al. New molecular markers for the evaluation of gamete quality. J Assist Reprod Genet. 2013;30(2):207-12.

75. Khalil A et al. Chromosome territories have a highly nonspherical morphology and nonrandom positioning. Chromosome Res. 2007;15(7):899-916.

76. Manuelidis L. A view of interphase chromosomes. Science. 1990;250(4987):1533-40.

77. Marshall WF. Order and disorder in the nucleus. Curr Biol. 2002;12(5):R185-92.

78. Oliver B, Misteli T. A non-random walk through the genome. Genome Biol. 2005;6(4):214.

79. Cremer $\mathrm{M}$ et al. Non-random radial higher-order chromatin arrangements in nuclei of diploid human cells. Chromosome Res. 2001;9(7):541-67.

80. Meaburn KJ, Misteli T. Cell biology: chromosome territories. Nature. 2007;445(7126):379-781.

81. Foster HA, Bridger JM. The genome and the nucleus: a marriage made by evolution. Genome organisation and nuclear architecture. Chromosoma. 2005;114(4):212-29.

82. Bridger JM et al. The non-random repositioning of whole chromosomes and individual gene loci in interphase nuclei and its relevance in disease, infection, aging, and cancer. Adv Exp Med Biol. 2014;773:263-79.

83. Verschure PJ. Positioning the genome within the nucleus. Biol Cell. 2004;96(8):569-77.

84. Elcock LS, Bridger JM. Exploring the relationship between interphase gene positioning, transcriptional regulation and the nuclear matrix. Biochem Soc Trans. 2010;38(Pt 1):263-7.

85. Galeraud-Denis I, Lambard S, Carreau S. Relationship between chromatin organization, mRNAs profile and human male gamete quality. Asian J Androl. 2007;9(5):587-92.

86. Greaves IK et al. Conservation of chromosome arrangement and position of the $\mathrm{X}$ in mammalian sperm suggests functional significance. Chromosome Res. 2003;11(5):503-12.
87. Barratt CL. Male infertility joins the translational medicine revolution. Sperm DNA: from basic science to clinical reality. Mol Hum Reprod. 2010;16(1):1-2.

88. Finch KA et al. Nuclear organization in human sperm: preliminary evidence for altered sex chromosome centromere position in infertile males. Hum Reprod. 2008;23(6):1263-70.

89. Haaf T, Ward DC. Higher order nuclear structure in mammalian sperm revealed by in situ hybridization and extended chromatin fibers. Exp Cell Res. 1995;219(2):604-11.

90. Hazzouri $\mathrm{M}$ et al. Genome organization in the human sperm nucleus studied by FISH and confocal microscopy. Mol Reprod Dev. 2000;55(3):307-15.

91. Ioannou D, Griffin DK. Male fertility, chromosome abnormalities, and nuclear organization. Cytogenet Genome Res. 2010;133(2-4): 269-79.

92. Ioannou D et al. Nuclear organisation of sperm remains remarkably unaffected in the presence of defective spermatogenesis. Chromosome Res. 2011;19(6):741-53.

93. Manvelyan $\mathrm{M}$ et al. Chromosome distribution in human sperm- - a 3D multicolor banding-study. Mol Cytogenet. 2008;1:25.

94. Millan NM et al. Hierarchical radial and polar organisation of chromosomes in human sperm. Chromosome Res. 2012;20:87587.

95. Zalenskaya IA, Zalensky AO. Non-random positioning of chromosomes in human sperm nuclei. Chromosome Res. 2004;12(2): 163-73.

96. Mudrak O, Tomilin N, Zalensky A. Chromosome architecture in the decondensing human sperm nucleus. J Cell Sci. 2005;118(Pt 19):4541-50.

97. Meyer-Ficca M, Muller-Navia J, Scherthan H. Clustering of pericentromeres initiates in step 9 of spermiogenesis of the rat (Rattus norvegicus) and contributes to a well defined genome architecture in the sperm nucleus. J Cell Sci. 1998;111(Pt 10): 1363-70.

98. Zalensky A, Zalenskaya I. Organization of chromosomes in spermatozoa: an additional layer of epigenetic information? Biochem Soc Trans. 2007;35(Pt 3):609-11.

99. Sbracia $\mathrm{M}$ et al. Preferential location of sex chromosomes, their aneuploidy in human sperm, and their role in determining sex chromosome aneuploidy in embryos after ICSI. Hum Reprod. 2002;17(2):320-4.

100. Ioannou D et al. Twenty-four chromosome FISH in human IVF embryos reveals patterns of post-zygotic chromosome segregation and nuclear organisation. Chromosome Res. 2012;20(4):447-60.

101. Olszewska M, Wiland E, Kurpisz M. Positioning of chromosome $15,18, \mathrm{X}$ and $\mathrm{Y}$ centromeres in sperm cells of fertile individuals and infertile patients with increased level of aneuploidy. Chromosome Res. 2008;16(6):875-90.

102. Finch KA et al. Nuclear organisation in totipotent human nuclei and its relationship to chromosomal abnormality. J Cell Sci. 2008;121:655-63.

103. Palmer DK, O’Day K, Margolis RL. The centromere specific histone CENP-A is selectively retained in discrete foci in mammalian sperm nuclei. Chromosoma. 1990;100(1):32-6.

104. Govin J et al. Pericentric heterochromatin reprogramming by new histone variants during mouse spermiogenesis. J Cell Biol. 2007;176(3):283-94.

105. Amaral A et al. The combined human sperm proteome: cellular pathways and implications for basic and clinical science. Hum Reprod Update. 2014;20(1):40-62.

106. Brykczynska $U$ et al. Repressive and active histone methylation mark distinct promoters in human and mouse spermatozoa. Nat Struct Mol Biol. 2010;17(6):679-87.

107. $\mathrm{Wu} \mathrm{F}$ et al. Testis-specific histone variants H2AL1/2 rapidly disappear from paternal heterochromatin after fertilization. J Reprod Dev. 2008;54(6):413-7. 
108. Kono $\mathrm{T}$ et al. Birth of parthenogenetic mice that can develop to adulthood. Nature. 2004;428(6985):860-4.

109. Sati L et al. Double probing of human spermatozoa for persistent histones, surplus cytoplasm, apoptosis and DNA fragmentation. Reprod Biomed Online. 2008;16(4):570-9.

110. Barda $\mathrm{S}$ et al. BRDT gene sequence in human testicular pathologies and the implication of its single nucleotide polymorphism (rs3088232) on fertility. Andrology. 2014;2(4):641-7.

111. Berkovits BD, Wolgemuth DJ. The role of the double bromodomain-containing BET genes during mammalian spermatogenesis. Curr Top Dev Biol. 2013;102:293-326.

112. Wu SF, Zhang H, Cairns BR. Genes for embryo development are packaged in blocks of multivalent chromatin in zebrafish sperm. Genome Res. 2011;21(4):578-89.

113. Jiang L et al. Sperm, but not oocyte, DNA methylome is inherited by zebrafish early embryos. Cell. 2013;153(4):773-84.

114. Potok ME et al. Reprogramming the maternal zebrafish genome after fertilization to match the paternal methylation pattern. Cell. 2013;153(4):759-72.
115. Vavouri T, Lehner B. Chromatin organization in sperm may be the major functional consequence of base composition variation in the human genome. PLoS Genet. 2011;7(4): e1002036.

116. Bourc'his D, Bestor TH. Meiotic catastrophe and retrotransposon reactivation in male germ cells lacking Dnmt3L. Nature. 2004;431(7004):96-9.

117. Hurst LD, McVean GT. Clade selection, reversible evolution and the persistence of selfish elements: the evolutionary dynamics of cytoplasmic incompatibility. Proc R Soc Lond Ser B Biol Sci. 1996;263(1366):97-104.

118. Normark BB. The evolution of alternative genetic systems in insects. Annu Rev Entomol. 2003;48:397-423.

119. Nahkuri S, Taft RJ, Mattick JS. Nucleosomes are preferentially positioned at exons in somatic and sperm cells. Cell Cycle. 2009;8(20):3420-4. 